\title{
Noise level of reverse motion gearing of gearbox
}

\section{Tomáš Oudrnický1, Elias Tomeh ${ }^{2}$}

Technical University in Liberec, Liberec, Czech Republic

${ }^{2}$ Corresponding author

E-mail: ${ }^{1}$ oudrnickytomas@seznam.cz, ${ }^{2}$ elias.tomeh@tul.cz

Received 30 April 2017; accepted 3 May 2017

DOI https://doi.org/10.21595/vp.2017.18564

Abstract. Quality requirements in the automotive industry are currently at an extremely high level; therefore, it is necessary to make various complex measurements, testing or observation of all important components of a vehicle whose integral part is a driving gear - a combustion engine and a gearbox. These components produce vibrations and noise, which means that it is produced by every current user of a passenger car. Particularly in a small-city-cars segment where such a big emphasis is not put on sound absorption of the cab for the crew. When taking into consideration this class of vehicles, it is important to prevent vibrations formation itself. These vibrations not only cause the unpleasant noise, but they also affect the durability of the surrounding components.

Keywords: noise level, vibrations, gearbox, gearing.

\section{Sources of noise of cogged gearboxes}

Each automobile gearbox which uses gearings for changing the gear ratio produces a certain noise level (Fig. 1). These days, the noise level of a gearbox is one of the important aspects of evaluation of the whole vehicle. It is a parameter which can be subjectively distinguished by every customer without any expertise. The noise level generally belongs among the most commonly occurring negative impacts on the product quality, not only in engineering. Vibration indication of an automobile gearbox is closely connected with the quality of the designed construction and the production accuracy.

The noise can be described quantitatively or qualitatively. Quantitative variables are those that can be measured (such as intensity, frequency, rhythm, etc.) and are defined by numeric values. Qualitative variables include subjective evaluation and are defined by different standards and other prescribed methodologies [1,2].

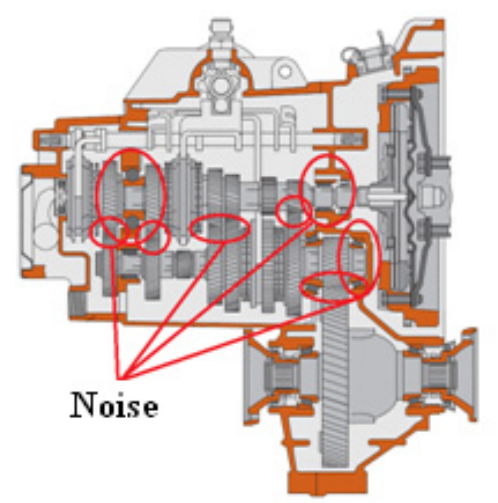

Fig. 1. Sources of noise in a gearbox [2]

\subsection{Noise from the cogwheels grip}

The main general reason of vibrations excitation from the gearing is the dynamic force which can be variable against its amplitude or place of action. The grip of the cogwheels does not consist only of the rolling itself, but also of spin. Therefore, the other frequent reason which was attached 
a great importance to in the past is vibrations excitation because of the existence of sliding speed. The vibrations from the grip are excited owing to the frequent changes of the sense of this sliding speed. Friction force dynamically changes when the grip is taking place. At first, it increases in one direction and after the rolling point is overcome - the shear force changes its sense - its size grows again. This feature is significant especially when taking into consideration the gear with a direct gearing.

In today's automobile gearboxes, gear sets with angular cogs which are at constant grip are exclusively used. When taking into consideration these gear sets, the vibrations which are produced owing to the influences of impacts from the cogs clearance of the free wheel are excited. By clearance it is meant a wheel placed on the shaft in a pivoted way. These impacts are also accompanied by impacts from the drive-axle assembly, so called "Disturbing noise" where the shaft is the subject to torsional oscillation. Therefore, flywheels are used to decrease the influences of these torsional oscillations $[1,2]$.

Parametric gearing excitation is directly influenced by the size of the coefficient of the grip duration $\varepsilon$. The advantage is that if this coefficient has an integral value (for example 2), a great change of grip rigidity will not appear because exactly two pairs of cogs will be in the grip all the time.

When taking into consideration the gearing of the automobile gearbox, a great attention should be paid to the constant gear - gearing - which connects the driven shaft of the gearbox with the differential gear. It occurs because this gear is loaded with torque whenever there is any change of gears. Therefore, its quality and service life should equate with it. The other gears which are currently without any load are affected only by passive resistances.

So, called tractive cog side and backward cog side are distinguished on each cogwheel. It means the side of the cog which is in gear when the vehicle is in traction, respectively when braking by the engine. Both sides can have differently defined parameters of gearing (for example $f_{h \alpha}, f_{h \beta}, C_{\alpha}, C_{\beta}$ ) because of the optimization of the starting conditions and decreasing the noise level of given gear both in traction and when braking by the engine.

The noise which appears when the cogwheels are in the grip is a significant source of noise in the whole gearbox. The gearing has a broad impact on the overall noise level of the gearbox. The following causes are presented in the production: tool geometry $\rightarrow$ tool adjustment in the machine $\rightarrow$ product (cogwheel) geometry $\rightarrow$ gearbox noise. When discovering that the noise level of the gearbox is above the set limit, the reverse process is analysed. For a correct reverse analysis, it is necessary to work correctly with the measured data. It is especially necessary to observe the tool, machine, material melting and hardening furnace which are currently being used. Today's automobile gearboxes use cogwheels with angular gearing which in practice cause approximately $5 \mathrm{~dB}$ lower noise than a direct gearing.

\subsection{Other sources of vibrations in a gearbox}

Among other parts which can cause noise most often belong different parts connected with changing gears (synchronizer rings, clutches, shift forks etc.)

Shifting mechanism belongs among the indirect sources of vibrations. If the shift fork stays in contact with a cogwheel, the vibrations will be transferred from the wheel into the whole shifting mechanism. Shafts transferring the torque are subjects to strain not only on torsion but considerably also on bending - their distortion, deflection and badly secured parallelism are also very often a reason of vibrations occurrence.

All those sources of noise transfer the created vibrations into the gearbox housing. Because a gearbox is a closed acoustic system, the gearbox noise is spread mostly by vibrations of the housing surface or by vibrations of connected components into the surroundings. Therefore, it also has a significant influence on the overall noise level of the gearbox. The value of the gearbox housing oscillation itself should not correspond with the discreet components of the gearing frequency, bearings or other parts which can be sources of dynamic excitation $[1,2]$. 


\section{Manifestations of noise level causes in vibration spectra}

Each source of noise in a gearbox has its specific manifestation in so called vibration spectra. For identifying the cause and the source of origin, different methods of technical diagnostics are used.

The spectrum can possibly be displayed as related to the individual shafts in a gearbox (SK-1, SK-2) or to the differential gear (SK-3), which enables closer determination of the source of the adverse noise. The "MIX" spectrum of the overall vibrations displays the sum of the three previously mentioned channels. Next, the spectra are marked as " $\mathrm{S}$ " (engine in acceleration) or "B" (engine braking).

\subsection{Manifestations of gearbox defects in vibration spectra}

Cogwheels produce combined spectrum of vibrations. Above the continuous part of the spectrum, individual discreet (harmonic) frequency components are highlighted. These components correspond with the frequency of grip of individual cogs - so called gear mesh frequency $\left(f_{Z}\right)$. In the Fig. 2, it is possible to see manifestations of basic defects of cogwheels in vibration spectra.

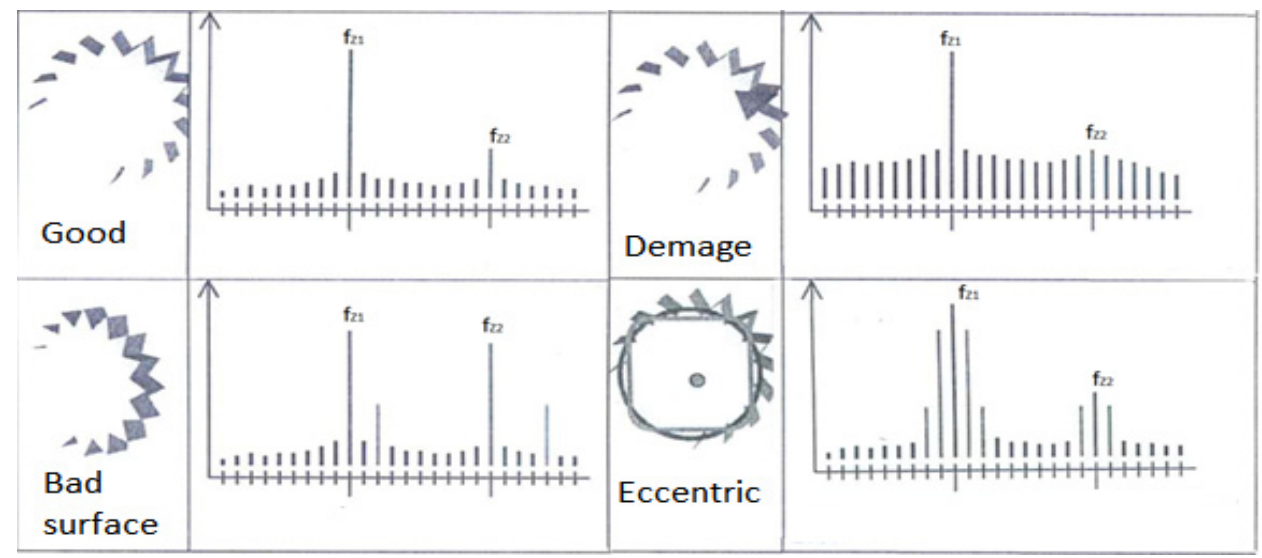

Fig. 2. Typical examples of noise from a cogwheel [3]

Involute (or generally gearing) wearing becomes evident by increasing the first $\left(f_{Z}\right)$ and the second harmonic gear mesh frequency $\left(2 \times f_{Z}\right)$. Higher orders of the harmonic frequency are caused by an advanced stage of damage or by another specific defect.

Anti-friction bearings become evident - in vibration spectra - by so called bearing frequency $\left(f_{L}\right)$. Its size depends on the geometry - bearing proportions, size of operating clearance and number of rolling bodies. We distinguish four bearing frequencies: outer ring frequency, inner ring frequency, cage frequency and rolling elements frequency.

Other sources of vibrations have their specific manifestations. For example, misalignment of shafts becomes evident by sidebands around gear mesh frequencies of gearing. Sideband frequencies are distant from $f_{Z}$ from each other about the value of a rotor frequency $\left(f_{R}\right)$ which is given by shaft frequency.

In the automobile gearbox there are more gearings which can - in spectra - also become evident, for example reverse motion gear set (which is the case of the gearbox).

\section{Noise level of reverse motion}

The reverse motion in a gearbox is realised by use of three cogwheels system. These cogwheels are equipped with straight conical cogs. From the motor, the torque is transferred through gearing 
on the drive shaft into the idle wheel of the reverse motion which is placed on an independent, stationary shaft. This gearing is in a constant grip. The last cogwheel of the reverse motion mechanics is a wheel which - when changing the gear - transfers the torque into the driven shaft of the gearbox and then through the driving system. This gearing, as the only one in a gearbox, is not in a constant grip.

The reverse gear is not provided with synchronization and the last mentioned gearing on the driven shaft is a part of the synchronizer sleeve of the fifth gear.

The interesting thing about this gear system is that the idle wheel of the reverse motion (green) is wider so that it could at the same time be in grip with two different cogwheels. Along its width there are two different segments of conical gearing (Fig. 3). The first segment of this wheel, with the angle $\beta=0^{\circ}$, is for the grip with the driving gearing on a shaft (blue). The second segment, with the angle $\beta=1^{\circ}$, is for the grip with the driven gearing on the clutch (red). This geometry results in pulling the wheel into the grip so that the "jumping-out" of the speed is avoided. This compound gearing is made by milling by use of an only tool [4].

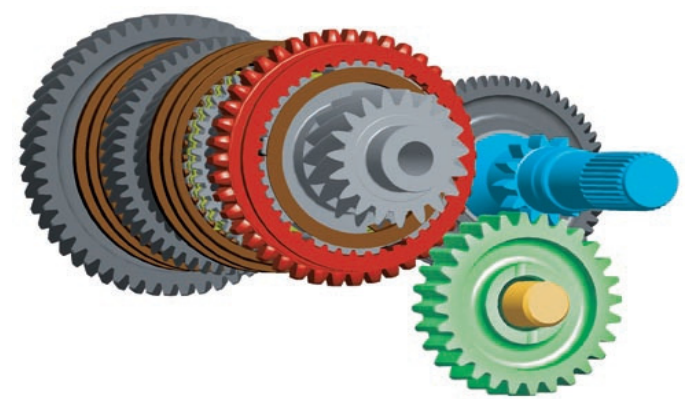

Fig. 3. Reverse motion gear [5]

\subsection{Comparison of a silent and a noisy gearbox}

For verifying the noise level of gearing, two tests were performed. During both tests, the same shaft and the same sliding clutch were used. Only the idle wheel was changed.

The green curve shows a silent gearing and the blue one shows a noisy gearing. The red curve shows the boundary values of the noise level.

First (Mix,KS)

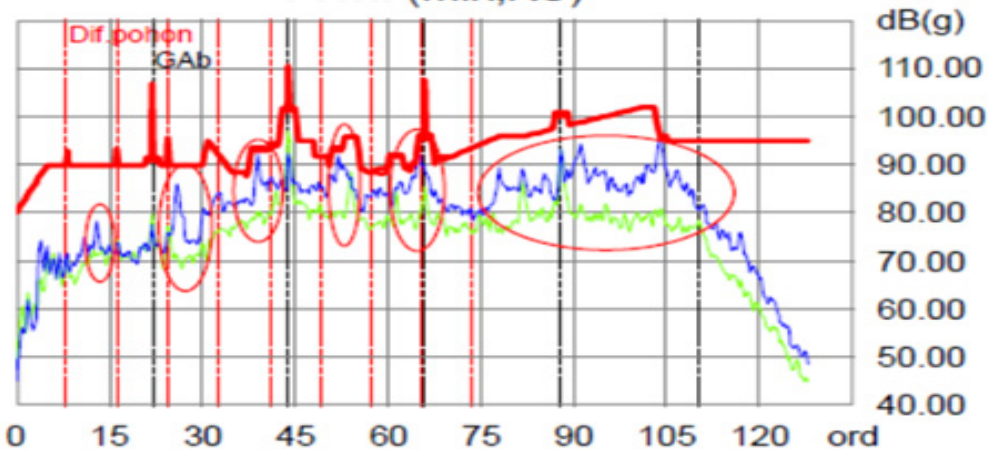

Fig. 4. Reverse motion gear set [1] 


\subsection{Difference on idle wheel}

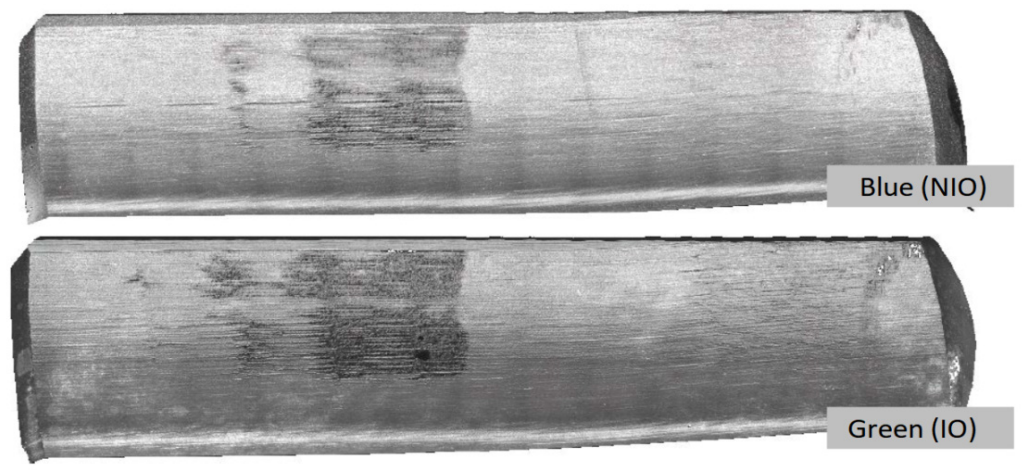

Fig. 5. Comparison of a trace after rolling on an idle wheel

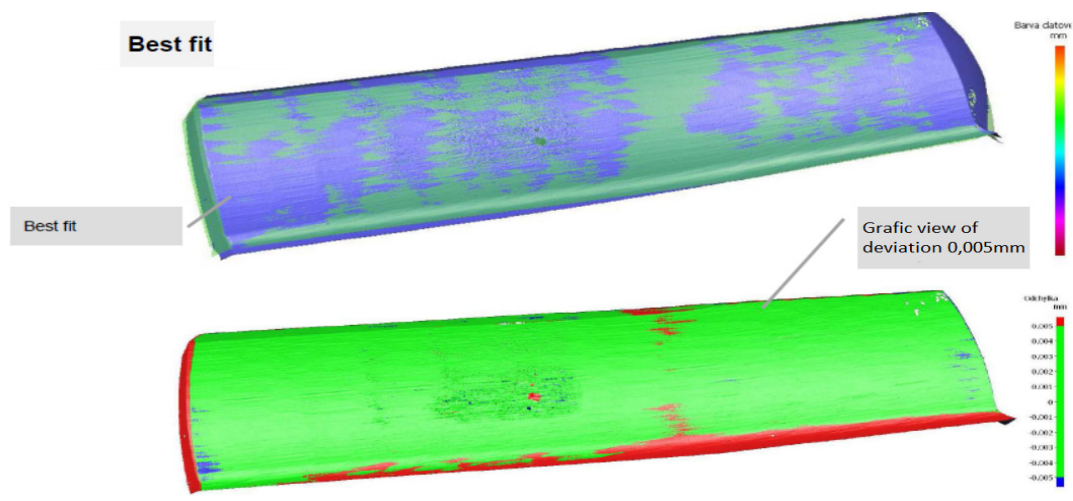

Fig. 6. Comparison of surface deviation

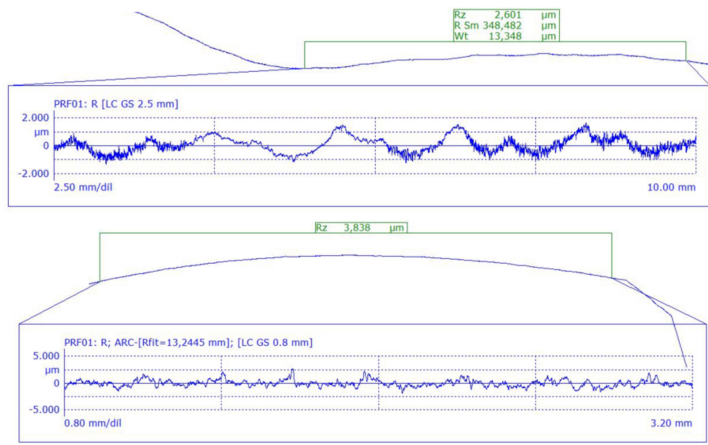

a) i.O Rz radial/axial

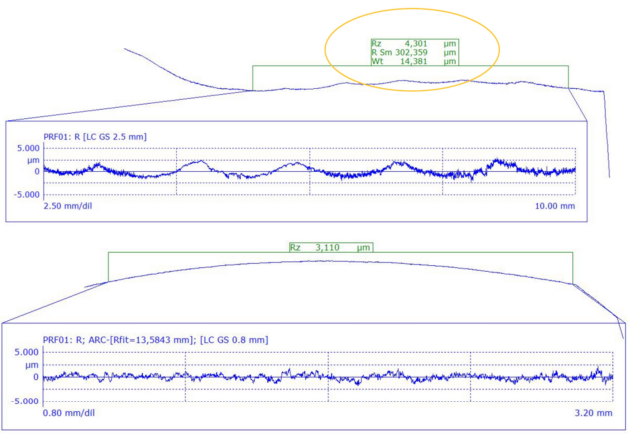

b) n.i.O Rz radial/axial

Fig. 7. Comparison of surface roughness

\section{Conclusions}

The idle wheel is quite a complex product because it consists of two different segments. Considering that, the wheel inclines to have production problems.

The protocol on gearing measurement was all right in both cases. It did not show any differences. Therefore, the analysis of other geometric proportions for discovering the cause of the increased noise level was performed. 

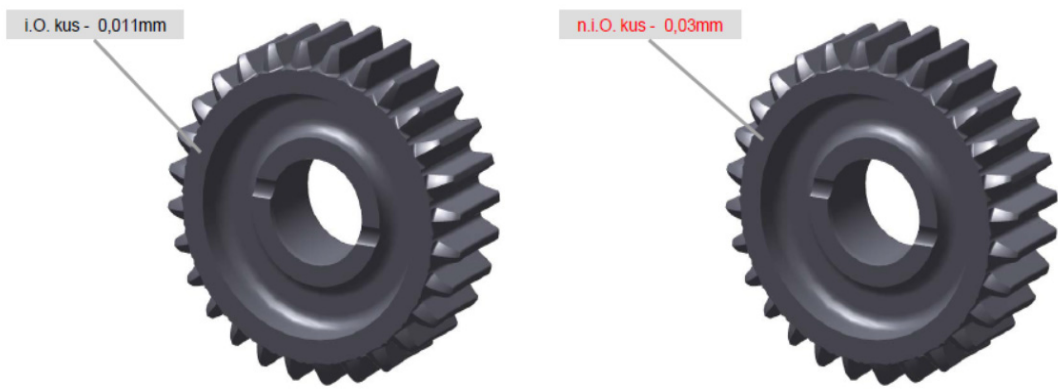

Fig. 8. Comparison of front runout value against its average

It appears from the metrological analysis of the idle wheel that even the smallest geometric deviations on the cogwheel can have a negative impact on the production of vibrations of gearing.

The noise level was characterised by a warble tone, which, in my opinion, happens because of the increased value of the front runout on the NIO part or also because of the increased value of roughness $R_{z}$.

After changing the cogwheel, the harmonic frequencies which correspond with the given gear set were decreased, the amplitude modulation was taken off and also the overall noise level was decreased. The manifestation corresponds with the presumption in accordance with the Fig. 2.

\section{References}

[1] Oudrnický T. Analysis of Causes of Noise in the Gearbox. Bachelor Thesis, Technical University in Liberec, 2014.

[2] Tomeh E. Vibration and Noise of Automobile Gear-Box in Connection with Identified Defects on Machine Tools. Technical university of Liberec, Liberec, 2008.

[3] Documents of Škoda Auto a.s., Mladá Boleslav, (Internal Data Source).

[4] Škoda Service, 5-speed Manual Transmission 0CF and Automated Five-Speed ASG Transmission. Workshop Teaching Aid No. 93, 2012.

[5] Tomeh E. Technical Diagnostics: Vibration Diagnostics of Machines and Equipment. Technical University of Liberec, Liberec, 2015.

[6] Tomeh E. Identify the sources of vibration and noise on cars gearbox by spectral analysis. 54th International Conference of Machine Design Departments, 2013.

[7] Oudrnický T. Constructional Modification of Gearbox for Noise Reduce. Diploma Thesis. Technical University of Liberec, Liberec, 2016. 